\title{
Eufemismos numéricos y conceptuales del paro español: cifras, fuentes y perfiles formativos
}

\begin{abstract}
Diana Amber
España se enfrenta a una alta cifra de desempleo que aún no ha recuperado los valores previos a la crisis económica. Sin embargo, las cifras no muestran todo el entramado de precariedad laboral que afecta a la sociedad española. Este trabajo parte de la concreción de la terminología usada en la medición del paro para estudiar la realidad referida al desempleo y a la precariedad laboral a través del análisis de estadísticas oficiales, descubriendo vacíos de información y silencios que suavizan los datos. Como estrategia metodológica se utiliza el análisis secundario de los informes de empleo de las fuentes oficiales españolas. Los resultados muestran cómo las diferentes conceptualizaciones intervienen en las cifras oficiales. Se ofrecen pistas para una interpretación más cercana a la realidad y se aterriza en la concreción del perfil formativo de la población desempleada. Se concluye que las cifras oficiales no reflejan con exactitud la vulnerabilidad social, obviando parte de la población en situación de riesgo, y que la formación ayuda, pero no es suficiente para ofrecer una respuesta absoluta al desempleo.
\end{abstract}

Palabras clave: Empleo. Desempleo. Formación. Exclusión.

\footnotetext{
*Universidad de Jaén (UJA), Jaén, España (damber@ujaen.es; https://orcid.org/0000-0002-9765-3547).
} 


\section{Justificación}

La precariedad laboral y el desempleo en España son un tema preocupante que alcanzó su máximo exponente durante la crisis económica, pero que años después del rebase de su punto álgido sigue atenazando a la población española. A pesar de los numerosos conflictos políticos, sociales y sanitarios acontecidos en los últimos tiempos, el desempleo sigue siendo una de las mayores preocupaciones sociales que hostiga a la población. Atendiendo a los barómetros de enero de los últimos cinco años del Centro de Investigaciones Sociológicas (CIS), el paro es señalado por aproximadamente el $60 \%$ de los encuestados como una de las principales problemáticas de la sociedad española, muy por encima de otras cuestiones de índole política o económica. Esta inquietud tan solo ha sido desbancada en los últimos meses por la preocupación por la pandemia y la crisis económica derivada de ella, aunque el desempleo persiste como preocupación para el 27,9\% de los encuestados en el barómetro de febrero de 2021 (CIS, 2021).

Según las últimas actualizaciones de la Oficina Europea de Estadística (Eurostat) España es actualmente el país europeo con mayor tasa de desempleo (EUROSTAT, 2021). El número de parados que registra el Servicio Público de Empleo Estatal en marzo de 2021 es de 3.949.640 (SEPE, 2021a), sin duda una alta cifra que, sin embargo, no representa al total de personas que se encuentran excluidas laboralmente o en una situación precaria o de vulnerabilidad social; personas que, como afirman Martínez-Martín, García-Moreno y Lozano-Martín (2018), están en riesgo de pobreza.

La huella de precariedad y marginación social y económica que la crisis dejó a nivel estatal y mundial en la población tras su arrasadora llegada es evidente (PEREIRA, 2015; SALAZAR, 2013). Sin embargo, y a pesar del fácil acceso a la información sobre desempleo, los datos facilitados a la ciudadanía son confusos. Los eufemismos impregnan discursos y cifras, puesto que se suavizan y maquillan muchos matices que hacen que los datos, a pesar de su dureza, sean más tolerables para la sociedad.

La principal contribución de este trabajo es aportar luz sobre los datos del desempleo, con la intención de dar a conocer la verdadera dimensión del paro y facilitar la lectura y la interpretación de los informes y estadísticas que la Administración pone a disposición del ciudadano.

\section{Marco conceptual}

A fin de evitar la ambigüedad en la interpretación de los datos, antes de adentrarse en las cifras es preciso partir de una aproximación conceptual que ayude a desentrañar la terminología utilizada por las dos fuentes estadísticas oficiales españolas para el tratamiento y la gestión de la información del desempleo. Estas fuentes son el SEPE y la Encuesta de Población Activa (EPA). 
El SEPE es un organismo adscripto al Ministerio de Trabajo, Migraciones y Seguridad Social cuyo fin es contribuir a la inserción y permanencia en el mercado de trabajo de las personas, así como a la mejora del capital humano de las empresas. Para ello, se encarga del desarrollo de la política de empleo y de la gestión del sistema de protección por desempleo (SEPE, 2020a). Este servicio elabora la estadística del paro de forma mensual, con base en los registros de las personas inscritas como demandantes de empleo en sus oficinas. La EPA es una investigación continua de las familias, cuya muestra inicial es de 65.000 familias por trimestre (aproximadamente doscientas mil personas). Se trata de un estudio estadístico que tiene como finalidad obtener información sobre el empleo y la situación laboral de los encuestados (INE, 2020). Esta estadística es elaborada de forma trimestral, por el Instituto Nacional de Estadística (INE).

En las cifras de los informes del desempleo presentados por ambas fuentes (EPA y SEPE) existen fuertes divergencias que, siguiendo a José Ignacio Pérez-Infante (2008, p. 53), «reflejan importantes diferencias de las dos estadísticas, tanto metodológicas como conceptuales y de colectivos considerados».

Adentrándonos en la terminología del SEPE, es preciso señalar que los términos demandante de empleo y desempleado que con frecuencia pueden ser interpretados indistintamente por la ciudadanía, son conceptos cercanos pero diferentes. En sus matices distintivos, se puede enmascarar un porcentaje de la población en situación de riesgo de exclusión, que no es contemplada como parada en los registros de esta fuente. Las personas demandantes de empleo son definidas por el SEPE (2020b, p. 1) como:

Trabajadores que se inscriben en los servicios públicos de empleo para la búsqueda de un empleo o mejorar el que ya poseen (intermediación laboral), para recibir otros servicios ajenos al empleo (orientación para el autoempleo, formación ocupacional...) o por la obligatoriedad de inscribirse para percibir una prestación contributiva o un subsidio.

La propia definición aclara que el término demandante de empleo incluye al desempleado, pero no se limita a este, ya que alberga también otra serie de condiciones profesionales diferentes al desempleo que, no por ello, implican una situación laboral estable o deseable. Por tanto, se considera como demandante de empleo a todo aquel que ejerce la solicitud de demanda, aunque no todos ellos formarán parte de las cifras del paro registrado presentes en los informes. Esto se debe a que la Orden Ministerial de 11 de marzo de 1985 por la que se establecen criterios estadísticos para la medición del Paro Registrado dispone que se excluyen de este registro todas aquellas demandas que al final del mes de referencia se encuentran en alguna de las siguientes situaciones:

- pluriempleo: personas que solicitan otro empleo compatible con el que tienen;

- mejor empleo: aquellos que, teniendo un empleo, se inscriben para lograr una mejora laboral;

- volaboración social: perceptores de prestaciones por desempleo que participan en trabajos de colaboración social; 
- jubilados;

- empleo coyuntural: personas que solicitan un empleo para un período inferior a tres meses;

- jornada inferior a veinte horas: aquellos que buscan un empleo con una jornada inferior a veinte horas semanales;

- estudiantes:

- demandantes que están cursando formación oficial reglada siendo menores de 25 años o que, superada esta edad, sean demandantes de primer empleo;

- demandantes asistentes a cursos de formación profesional ocupacional (con más de veinte horas lectivas a la semana, beca al menos de manutención y demandantes de primer empleo);

- demandas suspendidas;

- compatibilidad de prestaciones: beneficiarios de prestaciones por desempleo en situación de compatibilidad de empleo por realizar un trabajo a tiempo parcial;

- Trabajadores Eventuales Agrícolas Subsidiados (TEASS);

- rechazo de acciones de inserción laboral;

- otras causas: falta de disponibilidad inmediata, estar inscripto para participar en un proceso de selección para un puesto concreto, ser solicitante de trabajo exclusivamente en el extranjero o de empleo a domicilio, etcétera.

La inmensidad de exclusiones existentes para la pertenencia al colectivo de parados registrados, aun siendo demandante de empleo, es turbadora y preocupante, especialmente si consideramos la elevada cifra de desempleados registrados que, a pesar de las ingentes casuísticas que permiten excluirlos de este registro, siguen formando parte de los considerados parados. Actualmente, las diferentes situaciones laborales de los demandantes de empleo se agrupan en tres grandes bloques (SEPE, 2020c):

- ocupados: aquellos que ya tienen un empleo y buscan una mejora laboral u otro empleo con el que compatibilizar el que ya tienen;

- con disponibilidad limitada: personas sin empleo que en su solicitud especifican condiciones especiales de trabajo;

- demandantes de empleo no ocupados (DENOS): entre los que se encuentran los parados registrados y otros colectivos (denominados en los informes otros no ocupados) como estudiantes, trabajadores eventuales agrícolas subsidiados, etcétera.

Todas estas exclusiones del registro del paro suavizan las cifras creando la ilusión de una situación laboral más alentadora. Pues ni tan siquiera la totalidad de los denominados «demandantes de empleo no ocupados», engrosan las cifras oficiales del paro.

Atendiendo ahora a la terminología utilizada por la EPA, que sigue criterios internacionales definidos por la Organización Internacional del Trabajo (OIT) para clasificar 
a una persona como parada, la situación laboral de las personas puede clasificarse en una de las siguientes categorías (INE, 2020):

- activos: personas de 16 o más años que contribuyen a la producción de bienes y servicios o que están disponibles para incorporarse al sistema productivo. Se dividen en:

- ocupados: personas que trabajan (al menos una hora semanal) a cambio de una retribución en dinero o especie;

- parados: personas que se encuentran sin trabajo, pero disponibles para trabajar y en busca activa de empleo;

- inactivos: personas de 16 o más años que no se encuentran incluidas en ninguna de las categorías anteriores.

Cabe señalar en este punto cómo la simple inclusión en la definición de parado de la condición de que el sujeto se encuentre en búsqueda activa de empleo, logró un gran descenso de las cifras de parados de la EPA en el año 2000, hecho que coincide con la entrada en vigor del Reglamento de la Comisión Europea 1897/ 2000 que, de acuerdo a las normas aprobadas por la OIT en la decimotercera y decimocuarta Conferencia Internacional de Estadísticos del Trabajo (CIET), especifica que los desempleados se encuentren

[...] en busca de trabajo activa, es decir, que hayan tomado medidas concretas para buscar un empleo por cuenta ajena o un empleo por cuenta propia en un período de cuatro semanas que se termine al final de la semana de referencia. (CE, 2000, p. 19)

Este requisito en la consideración del término parado logra oportunamente que parte de la población que antaño se consideraba parada sea clasificada desde entonces como inactiva y, por lo tanto, disminuya la cifra final del desempleo aportada por la EPA.

Con relación a ambas fuentes de datos de desempleo (SEPE y EPA), es de suponer que, usando criterios diferentes para clasificar a la población, los resultados de ambas estadísticas no coincidan (PÉREZ-INFANTE, 2008). Entre otras razones, porque no todos los desempleados que cumplen los criterios de la OIT buscan empleo a través del SEPE y, en consecuencia, no todos ellos están inscriptos en dicho servicio, requisito imprescindible para ser parado registrado.

Esta aproximación conceptual pone de manifiesto la sencillez con la que la información cuantitativa puede ser modificada y cuestiona la objetividad de las cifras y su ajuste con las realidades sociales que estudian (AMBER; DOMINGO, 2017a). No es posible representar en cifras la dura realidad del mercado laboral actual, en el que la competitividad y la transitoriedad son notas fundamentales (LOMBANA-COY; MUÑOZ-SANTIAGO, 2017), y las desigualdades y vulnerabilidades laborales son perentorias a nivel global (DARI; FREGNANI, 2019; RECIO, 2018).

Es evidente que la probabilidad de acceso y mantenimiento de un empleo no es igual para todos. Numerosos factores como la edad, el sexo-género, la etnia-raza, el nivel formativo, etc., juegan un papel determinante (SUBIRATS, 2004) y pueden generar procesos de exclusión entre los más vulnerables (CASTEL, 2004). Estas situaciones de exclusión 
social, que tienen un carácter multidimensional, como apunta Salvador Manzanera-Román (2018), suponen el alejamiento progresivo de los espacios de integración social motivado por diferentes variables que afectan la vida de las personas -como, por ejemplo, el desempleo o la precariedad laboral-, limitando su acceso a los recursos de protección social. Ante esta problemática social el Real Decreto Legislativo 3/2015, de 23 de octubre, por el que se aprueba el texto refundido de la Ley de Empleo se propone entre sus objetivos generales «Asegurar políticas adecuadas de integración laboral dirigidas a aquellos colectivos que presenten mayores dificultades de inserción laboral, especialmente jóvenes, mujeres, personas con discapacidad y parados de larga duración, mayores de 45 años» (p. 6). De este modo, entendiendo que la formación puede mejorar las posibilidades de inserción laboral, la normativa vigente establece prioridades en la participación en acciones formativas de las personas más vulnerables, como queda recogido en el Real Decreto $694 / 2017$, de 3 de julio, por el que se desarrolla la Ley 30/2015, de 9 de septiembre, por la que se regula el Sistema de Formación Profesional para el Empleo en el ámbito laboral.

Sin embargo, los datos y las estadísticas no manifiestan si estas consideraciones legales están respondiendo a las necesidades laborales de los colectivos más vulnerables, las cifras del paro no atienden a casuísticas y la complejidad de su entramado no facilita su interpretación. La pertinencia de este estudio queda justificada en la necesidad de revisar los informes oficiales desde una mirada crítica que evidencie la existencia de estos puntos en blanco y lagunas informativas que tornan la información menos desalentadora.

\section{Marco metodológico}

La metodología utilizada para responder a los fines de este estudio es de carácter eminentemente cuantitativo, aunque con aspectos hermenéuticos que permitieron la interpretación de la información a partir del estudio conceptual ya presentado. En concreto, se hizo un análisis secundario de las estadísticas oficiales públicas. Este tipo de análisis libera los datos de la influencia y predisposición propia de la investigación primaria (ZÚÑIGA, 2011) y permite estudiar el fenómeno desde una nueva mirada (SCRIBANO; DE SENA, 2009), ayudando a su comprensión y respondiendo a nuevos interrogantes y propósitos, por lo que se considera especialmente pertinente para los fines de este artículo. Esta técnica permitió valorar la consistencia entre diferentes estudios que hacen referencia a la misma problemática, así como que emergieran datos que no aparecen representados con claridad en las estadísticas.

Las fuentes analizadas son los principales referentes nacionales para la medición del desempleo: el SEPE y la EPA. Los análisis se agruparon en tres acciones generales. El primero de ellos fue un estudio de contraste entre las cifras de desempleo ofrecidas por las diferentes fuentes oficiales analizadas. Esta comparación fue facilitada por el diseño y la aplicación del índice de discrepancia entre fuentes, que indica el porcentaje de discrepancia 
entre los datos de la EPA y del SEPE calculado a partir de la diferencia respecto al valor medio de las fuentes. Se puede establecer mediante la aplicación de la siguiente fórmula: ${ }^{1}$

$I D=\frac{\left(p^{E P A}-p^{S E P E}\right) 100}{\bar{X} p}$

Luego, en la segunda acción investigadora, se cruzó información cuantitativa contrastando la población identificada como parada por ambas fuentes con el total de personas que, agrupadas bajo otras denominaciones, pueden estar en riesgo de exclusión sociolaboral o en situación de precariedad. Finalmente, como tercera acción, se contrastó la información sobre la relación entre el nivel de formación y la tasa de desempleo ofrecida por ambas fuentes, definiendo el perfil general formativo del desempleado trazado por las estadísticas.

Se seleccionó como muestra una colección amplia de los resultados presentes en los informes de las dos fuentes seleccionadas, incluyendo los datos más recientes en el momento de realización del estudio. Para la primera acción investigadora se tomaron los informes de Demandantes de empleo, paro, contratos y prestaciones por desempleo de octubre, noviembre y diciembre de 2020 del SEPE (2021a) por ser los más recientes y coincidentes con el último informe trimestral de la EPA. También de analizaron esos mismos meses para los años 2018 y 2019 con el fin de apreciar la evolución temporal de los datos. En la segunda de las acciones, para observar la evolución temporal de los datos se utilizaron los informes oficiales de demandantes de empleo ofrecidos por el SEPE para el mes de enero desde 2006 hasta 2021 y los datos estadísticos de la EPA para el cuarto trimestre desde 2006 hasta 2020, con intención de incluir la información de mayor actualidad ofrecida por cada una de las fuentes. Los datos utilizados para el tercer análisis son los contenidos en la Estadística de demandantes de empleo, puestos de trabajo y colocaciones del SEPE (2021b) para octubre, noviembre y diciembre de 2020 y las cifras ofrecidas por la EPA para ese mismo trimestre.

\section{Resultados}

El análisis de contenido se ha dividido en tres temas de estudio que coinciden con las tres acciones de análisis antes señaladas, que dan estructura a la presentación de los resultados.

\section{Una realidad, diferentes interpretaciones numéricas}

Las cifras halladas al estudiar la situación del desempleo en la actualidad, además de fundirse en terminologías y propuestas taxonómicas complejas, difieren, como ya se ha comentado, entre las instituciones que las registran, lo que crea una panorámica difusa del desempleo.

${ }^{1} P^{E P A}=$ valor absoluto de parados según la EPA; $P S E P E=$ valor absoluto de parados según el SEPE; $\bar{X}_{p}=$ media de los valores absolutos de parados ofrecidos por la EPA y el SEPE. 
Con el fin de evidenciar las discrepancias, se usan los datos de desempleo de las 17 comunidades autónomas españolas, sus dos ciudades autónomas y los totales a nivel estatal. Cabe destacar dos cuestiones que dificultan el contraste de la información. Por una parte, las estadísticas ofrecidas por la EPA tienen carácter trimestral y las del SEPE, mensual, por lo que para su correcta comparativa se ha utilizado la media de parados calculada a partir de los datos mensuales ofrecidos por el SEPE para los meses analizados (octubre, noviembre y diciembre de 2020). Por otra parte, el SEPE no facilita la tasa de desempleo, puesto que gestiona datos y cifras de inscritos en el servicio, mientras que esta tasa sí es ofrecida por la EPA, que trabaja con datos poblacionales, por lo que, en ambos casos, se ha optado por analizar los valores absolutos para mejorar así la validez de la comparativa.

La Tabla 1 muestra las fuertes discrepancias halladas entre ambas fuentes en los datos del paro de las distintas comunidades a partir del cálculo de la diferencia entre sus valores absolutos en 2020 y del índice de discrepancia entre fuentes de ese mismo año y para 2019 y 2018 , lo que permite su comparación temporal. La presencia de resultados negativos y positivos evidencia la falta de sintonía entre ambas. Los valores por debajo de cero indican una mayor cifra de parados ofrecidos por el SEPE frente a los valores presentados por la EPA, pero la aparición aleatoria de valores positivos y negativos en función de la comunidad o incluso del año consultado dificulta establecer un patrón relacional entre las fuentes. La consulta más actual, del cuarto trimestre de 2020, muestra datos de paro más acusados por parte del SEPE, que supera las cifras de la EPA en trece comunidades y también a nivel nacional. Esta tendencia se suaviza en las consultas de años precedentes, en los que valores del desempleo marcados por el SEPE superaban a la EPA en once y en diez comunidades para los años 2019 y 2018 respectivamente.

Por su mayor porcentaje de discordancia entre fuentes, en 2020, destacan Asturias, Cantabria y País Vasco, con un índice de discrepancia de 29,9\%, 27,48\% y 26,86\% respectivamente, que mantiene la tendencia al alza visible en años anteriores, siendo en los tres casos el SEPE la fuente que muestra valores más altos de parados. El cuarto índice de discrepancia más elevado en 2020 lo presenta la comunidad de las Islas Baleares, con un $26,96 \%$, donde las cifras presentadas por la EPA son menos favorables que las del SEPE (como indica el valor positivo del índice).

Las mayores coincidencias entre ambas fuentes en 2020, representadas por los índices más bajos, se dan en Melilla, donde el índice de discrepancia es de 0,56\%, en la comunidad de Aragón con un 1,2\% y, curiosamente, a nivel estatal, como muestran los datos totales, tendencia a la baja que se muestra en mayor o menor medida también en años precedentes. Sin embargo, aunque el predominio de los valores al alza de una fuente u otra se mantiene habitualmente en la comparativa temporal, sorprende el giro de los datos nacionales, pues los valores de desempleo en 2018 y 2019 era mayores según la EPA y esta tendencia se invierte en 2020, tornándose superiores las cifras de parados del SEPE, lo que pone de manifiesto un incremento del número de parados registrados en el 
servicio de empleo durante el último año. Esta inversión de los datos se aprecia en también en comunidades concretas como Andalucía, Extremadura y Murcia.

TABLA 1

Valores absolutos del desempleo e índice de discrepancia entre fuentes Comunidades autónomas de España - 2018-2020

\begin{tabular}{|c|c|c|c|c|c|c|}
\hline \multirow[t]{2}{*}{ Comunidad autónoma } & \multicolumn{2}{|c|}{$\begin{array}{c}\text { Número de parados } \\
\text { (cuatro trimestre 2020) }\end{array}$} & \multirow{2}{*}{$\begin{array}{c}\text { Diferencia } \\
\text { (EPA-SEPE) } \\
\text { (1) }\end{array}$} & \multicolumn{3}{|c|}{$\begin{array}{l}\text { Índice de discrepancia entre fuentes } \\
(\%)\end{array}$} \\
\hline & EPA & SEPE & & 2020 & 2019 & 2018 \\
\hline Andalucía & 907.200 & 967.116 & -59.916 & $-6,39$ & 4,64 & 3,56 \\
\hline Aragón & 81.100 & 80.129 & 971 & 1,20 & 0,15 & 8,58 \\
\hline Principado de Asturias & 60.000 & 81.096 & -21.096 & $-29,90$ & $-16,97$ & $-23,48$ \\
\hline Islas Baleares & 109.900 & 83.794 & 26.106 & 26,96 & 7,87 & 18,35 \\
\hline Canarias & 279.000 & 266.748 & 12.252 & 4,49 & 3,58 & 9,09 \\
\hline Cantabria & 31.700 & 41.798 & -10.098 & $-27,48$ & $-16,76$ & $-34,65$ \\
\hline Castilla La Mancha & 173.000 & 184.413 & -11.413 & $-6,39$ & $-4,38$ & $-5,25$ \\
\hline Castilla y León & 128.300 & 163.659 & -35.359 & $-24,22$ & $-10,14$ & $-16,37$ \\
\hline Cataluña & 537.900 & 488.973 & 48.927 & 9,53 & 4,35 & 13,87 \\
\hline Com. Valenciana & 398.000 & 434.968 & -36.968 & $-8,88$ & $-3,98$ & $-6,78$ \\
\hline Extremadura & 104.700 & 110.864 & -6.164 & $-5,72$ & 14,26 & 9,89 \\
\hline Galicia & 142.600 & 186.122 & -43.522 & $-26,48$ & $-12,41$ & $-13,12$ \\
\hline Com. de Madrid & 480.000 & 430.907 & 49.093 & 10,78 & 2,73 & 13,63 \\
\hline Región de Murcia & 111.700 & 119.926 & -8.226 & $-7,10$ & 16,04 & 8,99 \\
\hline Com. Foral de Navarra & 36.700 & 39.691 & -2.991 & $-7,83$ & $-13,09$ & $-4,37$ \\
\hline País Vasco & 101.800 & 133.382 & -31.582 & $-26,86$ & $-20,34$ & $-20,46$ \\
\hline La Rioja & 16.300 & 18.869 & -2.569 & $-14,61$ & $-2,69$ & $-0,20$ \\
\hline Ceuta & 9.900 & 12.965 & -3.065 & $-26,81$ & $-8,80$ & $-24,89$ \\
\hline Melilla & 9.800 & 9.745 & 55 & 0,56 & $-0,89$ & 2,14 \\
\hline Total & 3.719 .800 & 3.855 .164 & -135.364 & $-3,57$ & 0,38 & 2,07 \\
\hline
\end{tabular}

Fuente: Elaboración propia a partir del INE (2021) y del SEPE (2021).

(1) Diferencia entre los valores absolutos del paro registrado según la EPA y el SEPE.

En definitiva, salvo puntuales coincidencias, las manifiestas discrepancias entre los datos contribuyen al desconcierto y a la dispersión de la información aportada por las fuentes estudiadas.

\section{Eufemismos conceptuales con resultados numéricos}

\section{Demandantes versus parados según el SEPE}

Centrándonos en las cifras ofrecidas por el SEPE (2021a), al comparar los datos totales de demandantes de empleo con los de parados registrados, la cantidad se reduce, según el informe de enero de 2021, más de tres millones y medio de personas (diferencia mucho más acentuada que en años anteriores). Los demandantes de empleo suman un total de 7.479.837 personas, dentro de quienes se encuentran los considerados no ocupados (DENOS) que se reducen a 4.255.550, al no incluir en esta categoría a los denominados ocupados ( 2.830 .665 personas), ni a los que presentan una disponibilidad 
limitada (393.622). Las cifras se suavizan aún más cuando consideramos que no todos los demandantes de empleo no ocupados (DENOS) figuran como parados registrados en el SEPE. De todos ellos, 291.197 personas más son consideradas otros no ocupados/ TEASS y tampoco están incluidas en los registros del paro. Finalmente, solo 3.964 .353 son consideradas como parados registrados de los casi siete millones y medio de demandantes de empleo que buscan una oportunidad laboral por este medio. El uso de esta taxonomía supone una diferencia de 3.515.484 personas entre las consideradas paradas y el número total de demandantes, es decir, un $47 \%$ de las demandantes no son calificadas como paradas, a pesar de que en muchos de los casos no tienen una actividad laboral o remunerada y su situación puede ser de precariedad o especialmente vulnerable. Por tanto, las cifras, con ayuda de eufemismos conceptuales, se ven mermadas con claridad y diluyen así una problemática social de mayor calado y profundidad.

Con el fin de mostrar una panorámica de estas discrepancias entre cifras desde una perspectiva temporal, se presenta el Gráfico 1, que compara demandantes de empleo totales con parados registrados y demandantes que no son considerados parados (valor calculado a partir de la diferencia entre las cifras de demandantes y parados en valor absoluto), utilizando los datos del mes de enero de cada año, desde 2006 hasta 2021.

\section{GRÁFICO 1}

Valores absolutos de demandantes versus parados registrados

España - 2006-2020

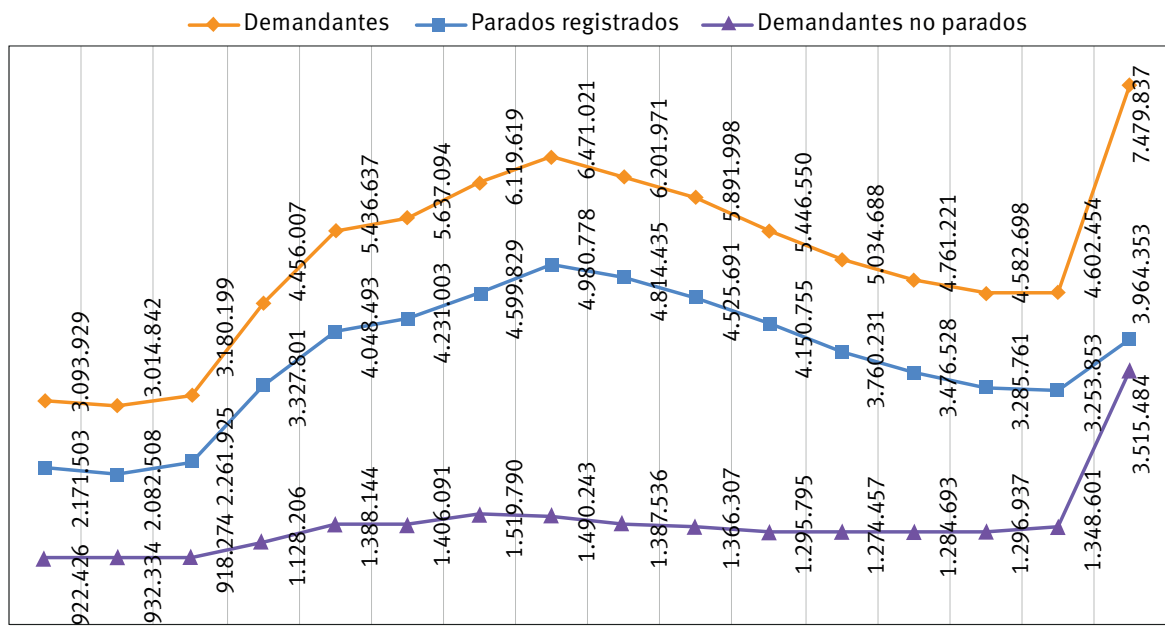

$2006200720082009201020112012201320142015 \quad 201620172018201920202021$

Fuente: Elaboración propia a partir del SEPE (2021a).

Como se puede ver en el Grafico 1, el número de demandantes de empleo es mucho mayor que el de los parados registrados, pues incluye también al resto de personas que, aun siendo demandantes, no figuran como desempleadas. Esta información se complementa con los datos de la Tabla 2, en la que se indica el número de personas demandantes (en valor 
absoluto y porcentual) que quedan al margen de las estadísticas de desempleo oficiales, así como el porcentaje de parados registrados respecto al total de demandantes entre 2006 y 2021, recogiendo los datos de los meses de enero (SEPE, 2021a).

TABLA 2

Diferencia entre demandantes y parados y porcentaje de demandates considerados parados registrados España - enero 2006-enero 2021

\begin{tabular}{cccc}
\hline Años & \multicolumn{2}{c}{ Demandantes no parados registrados } & $\begin{array}{c}\text { \% Demandantes parados } \\
\text { registrados (3) }\end{array}$ \\
\cline { 2 - 3 } 2006 & Valor absoluto (1) & 29,81 & 70,19 \\
2007 & 922.426 & 30,92 & 69,08 \\
2008 & 932.334 & 28,87 & 71,13 \\
2009 & 918.274 & 25,32 & 74,68 \\
2010 & 1.128 .206 & 25,53 & 74,47 \\
2011 & 1.388 .144 & 24,94 & 75,06 \\
2012 & 1.406 .091 & 24,83 & 75,17 \\
2013 & 1.519 .790 & 23,03 & 76,97 \\
2014 & 1.490 .243 & 22,37 & 77,63 \\
2015 & 1.387 .536 & 23,19 & 76,81 \\
2016 & 1.366 .307 & 23,79 & 76,21 \\
2017 & 1.295 .795 & 25,31 & 74,69 \\
2018 & 1.274 .457 & 26,98 & 73,02 \\
2019 & 1.284 .693 & 28,30 & 71,70 \\
2020 & 1.296 .937 & 29,30 & 70,70 \\
2021 & 1.348 .601 & 47,00 & 53,00 \\
\hline
\end{tabular}

Fuente: Elaboración propia a partir del SEPE (2021a).

(1) La diferencia entre el número de demandantes y el número de parados registrados indica el número de personas que aun siendo demandantes de empleo no son considerados parados (ocupados, con disponibilidad limitada, demandantes de empleo específicos, otros no ocupados/TEASS).

(2) Porcentaje de demandantes que pertenecen a otras categorías y, por tanto, no se consideran parados registrados por el SEPE.

(3) Porcentaje de demandantes que son considerados parados registrados según el SEPE.

Los datos de la Tabla 2 ponen de manifiesto que el porcentaje de parados respecto al total de demandantes creció durante el período de mayor intensidad de la crisis económica, mostrando su mayor índice en 2014, año en el cual 77,63\% de los demandantes eran considerados parados registrados y el $22,37 \%$ era excluido de esa categoría. El crecimiento de la proporción de parados durante la crisis económica respecto a la de demandantes de empleo, a pesar de las muchas excepciones que permiten a la administración reducir el número de parados (estudiantes, mejora laboral, disponibilidad limitada, empleo coyuntural, demandantes de primer empleo, etc.) y a los intentos de suavizar las estadísticas, muestra la situación crítica y la reducción de oportunidades que supuso esta crisis. En los últimos años anteriores a la llegada de la crisis sanitaria ocasionada por el coronavirus, la proporción estaba disminuyendo nuevamente, acercándose a los años previos al inicio de la crisis, aunque los valores absolutos del desempleo aún sumaban, en 2020, más de un millón de personas más que en los registros de 2006 (año anterior a la crisis económica), como se observa en el Grafico 1. Actualmente, en 2021, las cifras de 
demandantes de empleo dan un vuelco en la tendencia. Casi tres millones de personas más que en 2020 forman parte de los inscriptos como demandantes de empleo, una cifra superior a cualquier año precedente. Este aumento entre los inscriptos supone un incremento de más de dos millones de personas entre los demandantes no considerados parados registrados $y$, sin embargo, no genera una huella alarmante en los datos de desempleo, que no superan las cifras recogidas en los años centrales de la anterior crisis económica. De este modo, el acercamiento de las proporciones de parados registrados (53\%) y de aquellos que no son considerados oficialmente como tales ( $47 \%$ ) respecto al total de demandantes, deja entrever el aumento de personas que se encuentran actualmente en zona de vulnerabilidad, que pueden derivar en situaciones de exclusión laboral.

Inactivos y parados versus ocupados, según la EPA

La distribución de la población en función de su situación laboral a partir de los datos ofrecidos por la EPA para el cuarto trimestre de 2020 (INE, 2021) muestra un total de 23.064.100 personas consideradas activas, entre las que se incluyen las consideradas ocupadas (19.344.300 personas) y las paradas (3.719.800 personas), y, por otra parte, muestra una población inactiva compuesta por 16.571 .400 personas. Cabe recordar que entre la población de inactivos no solo están aquellas personas que no se encuentran en edad o situación de trabajar, sino también aquellos que, según los criterios de la EPA, no buscan empleo activamente.

Los datos ofrecidos por la EPA revelan que, dentro de la población activa, la mayor parte de las personas está ocupada y que el porcentaje de parados respecto a la población activa es poco mayor a $16 \%$, cifra elevada, especialmente en comparación con las tasas de desempleo del resto de los países europeos (EUROSTAT, 2021).

Al procesar los datos, hemos de recordar que el único grupo poblacional de los definidos por la EPA que contribuye a la producción de bienes o servicios es el de los ocupados. Teniendo esto en cuenta, en la Tabla 3 se presenta el sumario de la cifra de parados e inactivos y su diferencia respecto al número de ocupados, con base en las cifras aportadas por la EPA en el cuarto trimestre desde 2006 hasta 2020.

A partir de la Tabla 3 se elabora el Gráfico 2, que ilustra la tendencia temporal de los datos de ocupados, parados, inactivos y la sumatoria de los dos últimos, ofrecidos por la EPA para el período seleccionado.

Los datos de la Tabla 3, representados en el Gráfico 2, ponen de manifiesto cómo en 2006, año anterior a la crisis económica, había más de tres millones de personas ocupadas que las paradas e inactivas. Se observa claramente cómo durante los años centrales de la crisis el número personas que se encontraban dentro del sistema productivo era inferior a las que estaban al margen de este, como muestran los valores negativos de la última columna de la Tabla 3 para estos años. 
Relación de ocupados frente a inactivos y parados

España - 2006-2020

\begin{tabular}{cccccc}
\hline Años & Ocupados & Parados & Inactivos & $\begin{array}{c}\text { (parados + } \\
\text { inactivos) }\end{array}$ & $\begin{array}{c}\text { Diferencia } \\
\text { [ocupados - } \\
\text { (inactivos + } \\
\text { parados)] }\end{array}$ \\
\hline 2006 & 20.195 .400 & 1.819 .400 & 15.373 .800 & 17.193 .200 & 3.002 .200 \\
2007 & 20.717 .900 & 1.942 .000 & 15.441 .300 & 17.383 .300 & 3.334 .600 \\
2008 & 20.055 .300 & 3.206 .800 & 15.284 .500 & 18.491 .300 & 1.564 .000 \\
2010 & 18.890 .400 & 4.335 .000 & 15.487 .500 & 19.822 .500 & -932.100 \\
2011 & 18.674 .900 & 4.702 .200 & 15.424 .700 & 20.126 .900 & -1.452 .000 \\
2012 & 18.153 .000 & 5.287 .300 & 15.441 .500 & 20.728 .800 & -2.575 .800 \\
2013 & 17.339 .400 & 6.021 .000 & 15.422 .700 & 21.443 .700 & -4.104 .300 \\
2014 & 17.135 .200 & 5.935 .600 & 15.472 .300 & 21.407 .900 & -4.272 .700 \\
2015 & 18.569 .100 & 5.457 .700 & 15.496 .500 & 20.954 .200 & -3.385 .100 \\
2016 & 18.508 .100 & 4.237 .800 & 15.839 .000 & 20.076 .800 & -1.568 .700 \\
2017 & 18.998 .400 & 3.766 .700 & 15.951 .600 & 19.718 .300 & -719.900 \\
2018 & 19.564 .600 & 3.304 .300 & 16.150 .600 & 19.454 .900 & 109.700 \\
2019 & 19.966 .900 & 3.191 .900 & 16.268 .400 & 19.460 .300 & 506.600 \\
2020 & 19.344 .300 & 3.719 .800 & 16.571 .400 & 20.291 .200 & -946.900 \\
\hline
\end{tabular}

Fuente: Elaboración propia a partir de la EPA (INE, 2021).

GRÁFICO 2

Valores absolutos de ocupados, parados e inactivos

España - 2006-2020

$\multimap$ Ocupados $\longrightarrow$-Parados $\rightarrow$ Inactivos $\rightarrow$ Parados + inactivos

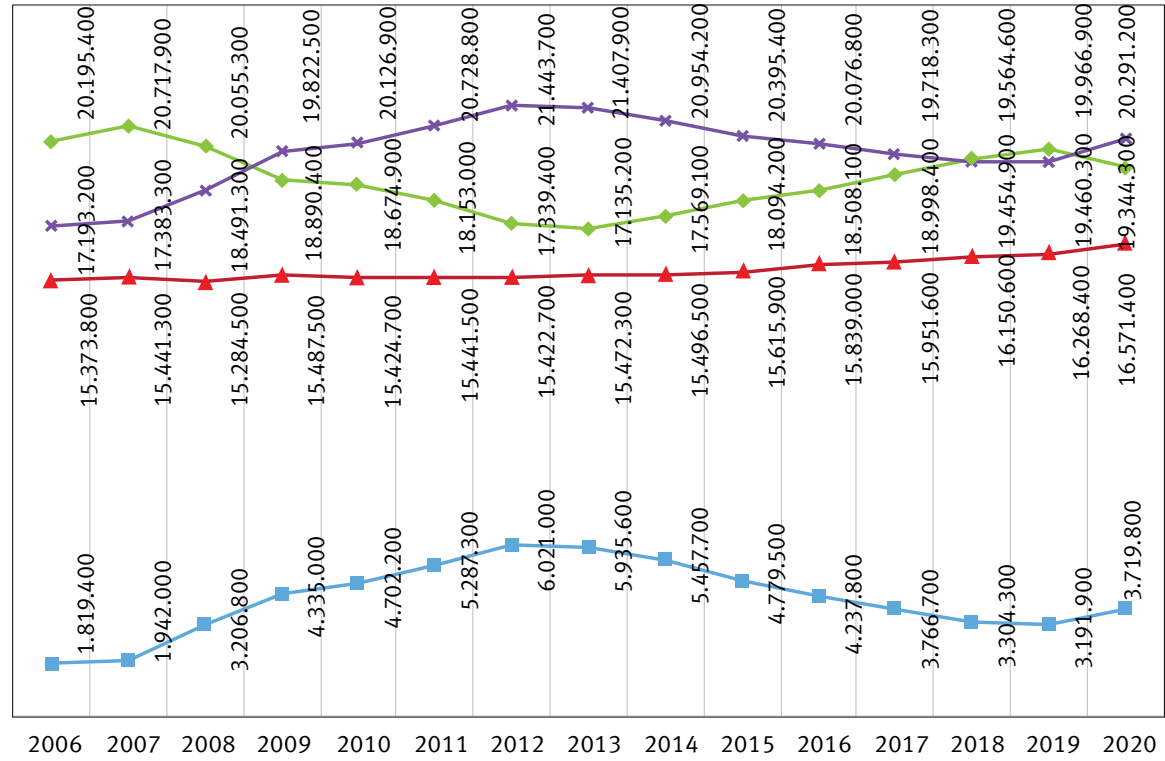

Fuente: Elaboración propia a partir de la EPA (INE, 2021). 
En especial en 2012 y en 2013 había más de cuatro millones de personas excluidas del sistema productivo que personas que estuviesen dentro de él. El número de ocupados, representado en el Gráfico 2 por la línea verde, vuelve a superar la cifra de parados e inactivos (línea morada) en los años 2018 y 2019, aunque levemente, tendencia que se invierte de nuevo en 2020 con la llegada de la crisis sanitaria y sus repercusiones en la economía y en el mercado laboral. También es destacable cómo el número de inactivos se está incrementando secuencialmente desde 2013, mientras que en años anteriores su crecimiento o descenso era intermitente, incremento que pueda asociarse probablemente en parte al envejecimiento de la población española.

\section{Formación versus empleo}

Los resultados del tercer análisis de este trabajo muestran los datos de desempleo en función del nivel de formación alcanzado por los desempleados a partir de las cifras de referencia aportadas por las dos fuentes oficiales analizadas (SEPE y EPA).

Nuevamente, para su contraste, ha sido necesario trabajar con la cifra media de los datos aportados por el SEPE para los meses de octubre, noviembre y diciembre de 2020, para contrastarlo con los datos trimestrales que ofrece la EPA para el mismo período. De igual forma, los niveles formativos se han agrupado en grandes categorías con el objetivo de contrastar los datos aportados por los informes de ambas fuentes. En la Tabla 4 se muestran las cifras de desempleo en función del nivel formativo aportadas por cada fuente en valores absolutos ( $y$ también porcentuales en el caso de la EPA, pues el SEPE no ofrece esta cifra) y el porcentaje respecto al total de la cifra de parados, indicando qué proporción de estos ha alcanzado cada uno de los niveles formativos.

TABLA 4

Registros de paro en función del nivel de formación España - cuarto trimestre de 2020

\begin{tabular}{lrrrrr}
\hline \multicolumn{1}{c}{ Estudios } & SEPE & EPA & \% & \multicolumn{2}{c}{$\begin{array}{c}\text { Porcentaje de parados } \\
\text { respecto al total }\end{array}$} \\
\cline { 5 - 7 } & & & & SEPE & EPA \\
\hline Sin estudios & 73.954 & 25.600 & 36,08 & 1,92 & 0,69 \\
Estudios primarios incompletos & 501.346 & 57.700 & 27,71 & 13,00 & 1,55 \\
Estudios primarios completos & 383.583 & 297.400 & 29,72 & 9,95 & 8,00 \\
Estudios secundarios & 2.320 .961 & 2.277 .500 & 18,51 & 60,20 & 61,2 \\
Estudios superiores & 575.318 & 1.061 .500 & 10,71 & 14,92 & 28,54 \\
Total & $\mathbf{3 . 8 5 5 . 1 6 4}$ & $\mathbf{3 . 7 1 9 . 8 0 0}$ & $\mathbf{1 6 , 1 3}$ & $\mathbf{1 0 0 , 0 0}$ & $\mathbf{1 0 0 , 0 0}$ \\
\hline
\end{tabular}

Fuente: Elaboración propia a partir de INE (2021) y SEPE (2021b).

Los valores absolutos aportados por las fuentes oficiales presentan de nuevo discrepancias entre ellos, pero en ambos casos alertan sobre la alta presencia de desempleados con estudios secundarios y superiores. Porcentualmente, más del $60 \%$ de los desempleados registrados por ambas fuentes han finalizado sus estudios secundarios, 
y entre el $14,92 \%$ y el $28,54 \%$, según la fuente analizada, presenta estudios superiores, mientras que la minoría de los parados registrados no tienen estudios o presentan estudios muy básicos. Sin embargo, atendiendo a la tasa de desempleo por nivel formativo ofrecida por la EPA, se observa que el $36,08 \%$ de la población activa sin estudios se encuentra en situación de desempleo.

Esta cuestión plantea dos lecturas, pues, aunque la tasa de desempleo es mucho mayor entre las personas con bajo nivel formativo (hecho que disminuye la empleabilidad), atendiendo a la situación formativa de la población parada, hay un porcentaje mayor de personas que tienen formación al menos secundaria frente a personas sin estudios o con estudios primarios, lo cual queda justificado por la mayor proporción de población activa que tiene estudios secundarios y superiores en España, frente a otros con niveles formativos inferiores, cada vez menos frecuentes en la población.

Por otra parte, cabe destacar que el SEPE registra un desempleo más precario a nivel formativo que el que recoge la EPA, incluyendo un mayor número de personas que presentan niveles educativos más bajos. Así, el SEPE registra, por ejemplo, 73.954 personas sin estudios, mientras que la EPA, tan solo 25.600 personas. Igual ocurre con los datos sobre las personas paradas con estudios primarios incompletos, que son 501.346 según el SEPE y tan solo 57.700 para la EPA. Esta tendencia se mantiene en todos los niveles formativos, salvo en el más alto: los registros de parados con estudios superiores indican una cifra de 575.318 personas en el SEPE, mientras que la EPA casi duplica esa cifra, registrando 1.061.500 personas con estudios superiores en situación de desempleo. Este hallazgo evidencia nuevamente el contraste entre las fuentes y promueve la reflexión sobre la nitidez de las cifras.

\section{Conclusiones}

El análisis de las fuentes y los datos que este descubre muestran la gran información disponible al respecto y su facilidad de acceso, lo que crea una ilusión de transparencia. Sin embargo, las trabas conceptuales, las taxonomías y los rígidos criterios de pertenencia a cada categoría encubren la información en clave terminológica y numérica dificultando su interpretación por parte de la ciudadanía.

El análisis secundario de los datos pone en evidencia cómo más de tres millones y medio de personas, sin ser consideradas paradas, buscan una oportunidad laboral o una mejora de su situación profesional en las oficinas de empleo españolas, lo que delata su situación de precariedad. Las cifras y los eufemismos conceptuales que las recrean dulcifican la realidad, mostrando una visión parcial y acicalada que sirve de contención social, como denuncian también los estudios de Diana Amber y Jesús Domingo (2017a) y de Hans Brachinger (2007). De este modo, este trabajo pone de manifiesto que las cifras de desempleo son claramente insuficientes para comprender lo que ocurre en el mercado de trabajo y que no es posible reducir realidades sociales a simples guarismos. 
La formación, entendida como puerta hacia el empleo (ZÚÑIGA; SORIANO, 2019), conserva en la actualidad su potencial para mejorar la empleabilidad de las personas, como dejan entrever las estadísticas. No obstante, debido a los, en general, altos niveles formativos de la población activa española en la actualidad (variable estrechamente relacionada con la edad de los sujetos debido a los cambios acontecidos en el sistema educativo español a lo largo de los años), la mayor parte de los parados tienen estudios secundarios o superiores, generando un desajuste entre formación y empleo (HERRERA, 2017). La formación no garantiza el empleo, pero aumenta la probabilidad de conseguirlo. El análisis del perfil formativo de los desempleados aporta pistas que pueden orientar las acciones formativas hacia la orientación laboral, el desarrollo de estrategias de búsqueda de empleo, la actitud proactiva, etc., para ayudar al desempleado a potenciar sus recursos, sin olvidar a los menos formados, que precisan iniciativas formativas desde un enfoque más integral o la acreditación de las competencias adquiridas mediante la experiencia (AMBER; DOMINGO, 2017b).

En definitiva, se espera que este trabajo aporte luz a la interpretación de los informes del desempleo en España, favoreciendo la comprensión del entramado conceptual y numérico y evidenciando la vulnerabilidad social que obvian las cifras y a la que la formación, por sí sola, no puede dar respuesta.

\section{Referencias}

AMBER, D.; DOMINGO, J. What statistics hide? Secondary analysis of unemployment in Spain. International Journal of Euro-Mediterranean Studies, v. 10, n. 2, p. 29-47, 2017a. Disponible en: https://emuni.si/wp-content/uploads/2019/11/2017-10-2_29-48.pdf.

AMBER, D.; DOMINGO, J. Desempleo y precariedad laboral en mayores de 45 años. Retos de la formación e implicaciones educativas. Revista Iberoamericana de Educación, v. 73, n. 1, p. 121-140, 2017b. DOI: https://doi.org/10.35362/rie731129.

BRACHINGER, H. W. Statistik zwischen Lüge und Wahrheit. AStA Wirtschafts-und Sozialstatistisches Archiv, v. 1, p. 5-26, 2007. DOI: 10.1007/s11943-007-0001-z.

CASTEL, R. Encuadre de la exclusión. In: KARSZ, S. (coord.). La exclusión: bordeando sus fronteras. Barcelona: Gedisa, 2004, p. 55-86.

CE - Comisión Europea. Reglamento 1897/2000 de la comisión, de 7 de septiembre de 2000, por el que se establecen disposiciones de aplicación del Reglamento (CE) 577/98 del Consejo relativo a la organización de una encuesta muestral sobre la población activa en la Comunidad por lo que respecta a la definición operativa de desempleo. Diario Oficial de las Comunidades Europeas, n. 228, p. 18-21, 8 sep. 2000.

CIS - Centro de Investigaciones Sociológicas. Barómetros. Depositados en banco de datos, 2021. Disponible en: 〈http://www.cis.es/cis/opencm/ES/11_barometros/depositados. jsp?pagina $=1 \&$ orden=1\&desc $=$ null $>$. Acceso en: 10 abr. 2021.

DARI, J.; FREGNANI, A. P. A reforma trabalhista em foco: desconstrução da proteção social em tempos de neoliberalismo autoritário. Educação \& Sociedade, v. 40, p. 1-18, 2019. Disponible em: http://www.scielo.br/pdf/es/v40/1678-4626-es-40-e0223441.pdf. 
ESPAÑA. Orden Ministerial, de 11 de marzo de 1985, por la que se establecen criterios estadísticos para la medición del Paro Registrado. Boletín Oficial del Estado, n. 63, p. 6612, 14 mar. 1985.

ESPAÑA. Real Decreto Legislativo 3/2015, de 23 de octubre, por el que se aprueba el texto refundido de la Ley de Empleo. Boletín Oficial del Estado, n. 255, p. 1-29, 24 oct. 2015.

ESPAÑA. Real Decreto 694/2017, de 3 de julio, por el que se desarrolla la Ley 30/2015, de 9 de septiembre, por la que se regula el Sistema de Formación Profesional para el Empleo en el ámbito laboral. Boletín Oficial del Estado, n. 159, p. 56864-56899, 5 jul. 2017.

EUROSTAT. Unemployment rate by sex, 2021. Disponible en: https://appsso.eurostat.ec.europa. eu/nui/submitViewTableAction.do. Acceso en: 10 abr. 2021.

HERRERA, D. Empleabilidad versus sobrecualificación. Desajuste entre formación y empleo en las trayectorias laborales de los jóvenes titulados en España. Sociología del Trabajo, n. 89, p. 29-52, 2017.

INE - Instituto Nacional de Estadística. Sección prensa / Encuesta de Población Activa (EPA), 2020. Disponible en: https://www.ine.es/prensa/epa_prensa.htm. Acceso en: 10 feb. 2020.

INE - Instituto Nacional de Estadística. Encuesta de Población Activa (EPA), 2021. Disponible en: https://www.ine.es/dyngs/INEbase/es/operacion.htm?c=Estadistica_C\&cid=1254736176918\& menu=resultados\&secc=1254736195129\&idp=1254735976595. Acceso en: 10 abr. 2021.

LOMBANA-COY, J. E.; MUÑOZ-SANTIAGO, A. E. Competitividad, educación y empleabilidad: consideraciones para el desarrollo de los países. Clío América, v. 11, n. 22, 2017. DOI: https:// doi.org/10.21676/23897848.2437.

MANZANERA-ROMÁN, S. Las políticas públicas del mercado de trabajo como factor de intensificación de la precariedad laboral y la exclusión social en España. Prisma Social - Revista de Ciencias Sociales, n. 23, p. 332-352, 2018.

MARTÍNEZ-MARTÍN, R.; GARCÍA-MORENO, J. M.; LOZANO-MARTÍN, A. M. Trabajadores pobres en España. El contexto de la crisis económica como marco para comprender la desigualdad. Papeles de Población, v. 24, n. 98, p. 185-218, 2018.

PEREIRA, N. C. O impacto da crise econômica espanhola na mão de obra imigrante. Revista Brasileira de Estudos de População, v. 32, n. 1, p. 7-23, 2015. Disponible en: https://rebep.org. br/revista/article/view/692.

PÉREZ-INFANTE, J. I. ¿Por qué el paro registrado supera al paro de la EPA? Revista del Ministerio de Trabajo y Asuntos Sociales, n. 71, p. 53-65, 2008. Disponible en: https://dialnet.unirioja.es/ servlet/articulo? codigo $=2579654$.

RECIO, A. Desigualdades en el mercado laboral. In: MIGUÉLEZ, F. (coord.). La revolución digital en España. Impacto y retos sobre el mercado de trabajo y el bienestar. Bellaterra: Universitat Autònoma de Barcelona, 2018. p. 188-211. Disponible en: https://ddd.uab.cat/record/190324.

SALAZAR, B. ¿Crisis después de la crisis? La macroeconomía financiera después de la crisis global. Revista de Economía Institucional, v. 15, n. 28, p. 127-158, 2013.

SCRIBANO, A.; DE SENA, A. Las segundas partes sí pueden ser mejores: algunas reflexiones sobre el uso de datos secundarios en la investigación cualitativa. Sociologias, n. 22, p. 100-118, 2009. DOI: https://doi.org/10.1590/S1517-45222009000200006.

SEPE - Servicio Público de Empleo Estatal. Definición - Misión, visión, valores, 2020a. Disponible en: https://www.sepe.es/HomeSepe/que-es-el-sepe/que-es-el-sepe/mision-vision-valores. html. Acceso en: 10 feb. 2020. 
SEPE - Servicio Público de Empleo Estatal. Introducción y descripción de conceptos, 2020b. Disponible en: https://www.sepe.es/HomeSepe/que-es-el-sepe/estadisticas/empleo/ estadisticas-nuevas/2020/enero.html. Acceso en: 20 ene. 2020.

SEPE - Servicio Público de Empleo Estatal. Conceptos, 2020c. Disponible en: https://www. sepe.es/HomeSepe/que-es-el-sepe/estadisticas/datos-avance/conceptos.html. Acceso en: 20 ene. 2020.

SEPE - Servicio Público de Empleo Estatal. Resumen datos estadísticos, 2021a. Disponible en: https://www.sepe.es/HomeSepe/que-es-el-sepe/estadisticas/datos-avance.html. Acceso en: 10 abr. 2021.

SEPE - Servicio Público de Empleo Estatal. Datos estadísticos de empleo, 2021b. Disponible en: https://www.sepe.es/HomeSepe/que-es-el-sepe/estadisticas/empleo/estadisticas-nuevas. html. Acceso en: 11 abr. 2021

SUBIRATS, J. Pobreza y exclusión social. Un análisis de la realidad española y europea. Barcelona: Fundación La Caixa, 2004.

ZÚÑIGA, R. M.; SORIANO, E. Desempleo juvenil y programas empleo en Andalucía: ¿la formación como elemento clave? Revista de Ciencias Sociales, v. 25, n. 4, p. 22-39, 2019.

ZÚÑIGA, G. Retos técnicos y oportunidades del análisis secundario de datos. Ciencia-UANL, v. 14, n. 2, p. 132-136, 2011.

\section{Sobre la autora}

Diana Amber es Doctora en Fundamentos del Currículum y Formación del Profesorado en las Áreas de Primaria y Secundaria por la Universidad de Granada. Profesora contratada Doctora del Área de Teoría e Historia de la Educación de la Universidad de Jaén. Miembro de la Red RIDIPD.

\section{Dirección para correspondencia}

Departamento de Pedagogía

Campus Las Lagunillas, $\mathrm{s} / \mathrm{n}$

23071 - Jaén, España

\section{Resumo}

Eufemismos numéricos e conceptuais do desemprego espanhol: números, fontes e perfis formativos

A Espanha enfrenta uma alta taxa de desemprego e ainda não recuperou os patamares prévios à crise econômica. Não obstante, os números não mostram a trama de precariedade laboral que afeta a sociedade. 0 presente estudo parte da concretização da terminologia usada na medição do desemprego, para estudar a realidade referida ao desemprego e à precariedade laboral por meio da análise de estatísticas oficiais, descobrindo lacunas de informação e silêncios que suavizam os dados. Como estratégia metodológica é utilizada a análise secundária dos informes de emprego das fontes oficiais espanholas. Os resultados mostram como as diferentes conceptualizações intervêm nos números oficiais. Oferecem-se pistas para uma interpretação mais próxima no que 
se refere ao perfil formativo da população desempregada. Conclui-se que os números oficiais não refletem a vulnerabilidade social, ignorando parte da população em risco, e que a formação ajuda, mas não é suficiente para oferecer uma resposta absoluta ao desemprego.

Palavras-chave: Emprego. Desemprego. Formação. Exclusão.

\begin{abstract}
Numerical and conceptual euphemisms for Spanish unemployment: numbers, sources and training profiles

Spain deals with a high unemployment rate that has not yet recovered the values prior to the economic crisis. However, this does not show the grid of job insecurity that affects society. This study starts from the conciseness of the terminology used to quantify unemployment, to study the reality regarding unemployment and job insecurity through an analysis of official statistics given the lack of information and silence that obscure the data. As a methodological strategy, the secondary analysis of unemployment reports provided by Spanish official sources is used. Results show how different conceptualizations affect official figures. Clues are offered for a closer interpretation of reality grounded on the conciseness of a training profile of the unemployed people. It is concluded that official figures do not accurately represent social vulnerability, ignoring part of the population at risk, and that, although training does help, it is not enough to offer a comprehensive response to unemployment.
\end{abstract}

Keywords: Employment. Unemployment. Training. Exclusion.

Recebido para publicação em 18/03/2020 Aceito para publicação em 31/05/2021 